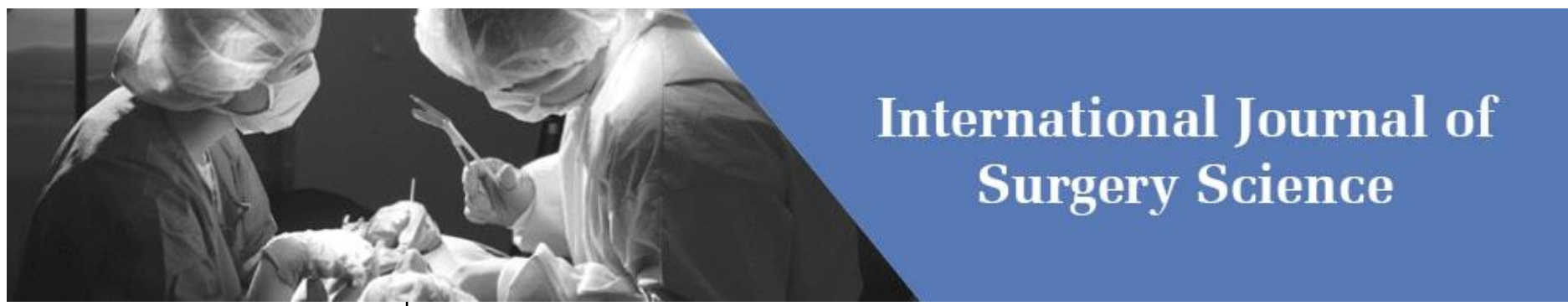

E-ISSN: 2616-3470

P-ISSN: 2616-3462

(C) Surgery Science

www.surgeryscience.com

2019; 3(4): 335-337

Received: 16-08-2019

Accepted: 20-09-2019

Dr. Surya Kumar Singh

Consultant Urologist, Balrampur

Hospital, Lucknow, Uttar Pradesh, India

Dr. Akhilesh Chandra

Consultant Urologist, Balrampur

Hospital, Lucknow, Uttar Pradesh, India
Corresponding Author:

Dr. Akhilesh Chandra

Consultant Urologist, Balrampur

Hospital, Lucknow, Uttar Pradesh,

India

\section{Place of diagnostic cystourethroscopy in evaluation of lower urinary tract symptoms (LUTS) and associated pathologies, experience of a district hospital}

\author{
Dr. Surya Kumar Singh and Dr. Akhilesh Chandra
}

DOI: https://doi.org/10.33545/surgery.2019.v3.i4f.265

\section{Abstract}

Background: Cystourethroscopy is used to diagnose and monitor conditions affecting bladder and urethra. LUTS i.e. lower urinary tract symptoms is a term used to describe a plethora of conditions involving bladder, sphincter, prostate and urethra, symptoms like hesitancy, urgency, frequency, weak stream, dribbling, etc. Collectively comprises LUTS. Symptoms point towards the cause of the problem viz urinary tract infection, prostatitis, Benign Prostatic Hyperplasia, Overactive bladder etc.

Objective: To study the value of Cystourethroscopy in a plethora of Urological disorders including cases of Haematurea, LUTS, UTI, incontinence etc.

Material \& Methods: This study was conducted at Balrampur Hospital, Lucknow, Urology department between 2014-2018, patients selected were between 18 yrs to 80 yrs of age, from both sexes. It is a retrospective uncontrolled study done on 1252 patient.

Patients were subjected to thorough examination including History and Clinical examination. Patients were selected having voiding symptom (both storage and obstructive), gross or microscopic Haematurea, evaluation of urologic fistulas, urethral or bladder diverticulum, incontinence patients, evaluation after prolapse procedures, bladder stones etc. Cystourethroscopy is done with a 17Fcystoscopesheath under local anesthesia and takes about 15 minutes. Patients having prior Urinary tract infection are treated with antibiotics before they are subjected to cystoscopy. There are hardly any complications related to the procedures, other than occasional transient minimal Haematurea, pain, occasional short episode of fever \& dysuria.

Results: Cystoscopy is a minor procedure without any major complications other than very trivial once like dysuria, mild pain. It's a definitive diagnostic tool which settles the diagnosis in cases such as strictures, $\mathrm{BPH}$, High bladder neck, stones, diverticulum, tumor or polyp, cases of fistulas, cystitis and helps in exclusion of cause of neurogenic bladder and overaction bladder. Hence it's a boon for patients of LUTS as far as settling the diagnosis is concerned.

Conclusion: Cystoscopy is a very important diagnostic tool for arriving at a definite diagnosis in cases of patients coming with Lower Urinary tract symptoms and other pathologies related to bladder, prostate and urethra.

Keywords: LUTS, cystourethroscopy, haematurea, BPH, strictures, diverticulum, bladder tumor

\section{Introduction}

Credit for early suggestion of fiberscope urethroscopy goes to Marshall in 1964. TSU Chida and Sugawara in 1973 used cystoscopy to examine bladder neck.

Cystourethroscopy is a very minor outdoor procedure which can be done under Local Anaesthesia. Most of the Lower urinary tract pathologies involving bladder, lower end of ureter i.e. UV junction, Bladder Neck, prostatitis\& urethra can be diagnosed with this simple procedure. BPH, Cystitis, stones, strictures. Malignancy, Diverticulum of both urethra \& bladder, false passages (iatrogenic), Neurogenic bladder can all be diagnosed. Cystoscopes use optical fibres and consist of lenses like telescope with an eyepiece at one end and tiny lens at the other end.

Patients were categorised according to their symptoms. LUTS (irritative and voiding), Haematurea, and Incontinence etc. Haematurea cases could be both having microscopic (UTI, Glomerulonephritis, nephropathy) or macroscopic (Bladder tumor) for purposes of diagnosis and follow up for recurrence following tumor resection (TURBT). 
Chronic pelvic pain syndrome and recurrent UTI. Often a cystoscopy may be found to be normal but it certainly helps in ruling out other cause of symptoms. Cystoscopy is also done for unusual cells found in Urine sample. Certain add-on procedures over and above cystoscopy can be of therapeutic value as well for taking Biopsies from bladder or excision of small polyp \&tumors, DJ stenting, collection of urine sample from Individual kidneys in cases of infection, RGP (dye injected into ureter \&x-raytaken). We have not gone into the therapeutic part in this paper.

\section{Material and Methods}

This study was conducted in Balrampur Hospital, Lucknow by Urology Department between 2016-2018 and 1252 patient were taken up for the study, aged between $18 \mathrm{yrs}$ to $80 \mathrm{yrs}$ of both sexes.

It is a retrospective uncontrolled study. Proper informed consent was taken before the procedure.

\section{Inclusion criteria}

1. Age between $18 \mathrm{yrs}$ and $80 \mathrm{yrs}$

2. Both sexes were included.

3. Patients suffering from LUTS (Both irritative and obstructive), Haematuria (both microscopic and macroscopic) incontinence (Neurogenic bladder, stress / urge incontinence, $\mathrm{OAB}$ ) and recurrent infections (stones, fistulas, obstruction).

\section{Exclusion criteria}

1. Patient having urinary infection

2. Patient with known bleeding disorder and uncontrolled coagulopathies

3. Any Medical contraindication

4. Uncooperative patient

We used rigid $17 \mathrm{~F}$ cystoscope sheath, a bridge and 30 degree fiber optic telescope for the examination.

Patients were subjected to History taking, Clinical examination both general and local and Pelvic examination including a DRE wherever indicated (after consent). Selected patients were asked to get viral Markers, CBC, Kidney Function test, Urine examination (Routine\& $\mathrm{M} / \mathrm{E}$ ) before being taken up for cystourethroscopy.

Patients were asked to come following morning after taking morning snacks and were taken into OT (after written informed consent).

We did not use prophylactic antibiotic in routine other than for immunocompromised patients, like Diabetics \& patients with recurrent UTI etc.

Patient is put in lithotomy and part preparation done with antiseptic lotion including betadine, draping done and lidocaine / xylocaine jelly introduced in urethra. Urethral opening clamped with penile clamp for few minutes for local anesthesia to take effect.

Cystourethroscope introduced through external urinary meatus and into the urethra thus ruling out any meatal stenosis or narrowing, xerotica obliterans, any stricture, diverticulum, urethral calculus can be seen. Cystoscope on passing through bulb is negotiated through membranous urethra, function of external sphincter can be elicited by asking the patient to stop urine, further ahead prostate, prostatic urethra, veru, bladder neck are visualized. Once inside the bladder trigone, uretric orfices and all walls of urinary bladder are thoroughly examined. Pathology like BPH, Prostatitis, stones, cystitis, bladder tumors or benign growth or haemangiomatous areas can be identified. Efflux from individual uretric orifice can show haematurea or chylous efflux as in cases of filarial chyluria.

Cystourethroscopy takes about 15 minutes in all. Patient can go back after passing urine. Few of the patients report minor complications like voiding dysuria, mild haematurea or frequency following cystoscopy and they are all self limiting. There is hardly any risk of upper tract infection. Patient on discharge is advised to take plenty of fluids orally and advised to report back to us in case of severe dysuria, rigors or fever.

\section{Results}

We had selected 1252 patients, both sexes, aged between 18 years to 80 years. They were selected based on inclusion/exclusion criteria already described. They were subjected to routine investigations like CBC, Viral Markers, urine test followed by Cystourethroscopy under Local anesthesia. We came across 30 patients who had meatal stenosis and it was not possible to negotiate cystoscope without making a dorsal cut in the meatus. i.e. $9 \%$ of patients.

We came across 150 patients (both sexes) who had stricture i.e. $12 \%$ of all patients. Of these 134 patients $(89 \%)$ had anterior stricture i.e. (Pendulous, bulbar urethra) and only 16 patients (11\%) had posterior stricture. Of these 150 stricture patients, 141 were male i.e. $94 \%$ and 9 were female i.e. $6 \%$.

In our study of 1252 patients, we came across 50 patients who had stone in there lower Urinary Tract (Bladder, prostatic Urethra, penile urethra). This contributed to around $4 \%$ of total no. of patients. Of these 50 stone cases 45 were bladder stones (i.e. $90 \%$ ), 3 were impacted in prostatic urethra i.e. $6 \%$ and 2 were impacted in penile urethra just proximal to Fossa Navicularis (i.e. 4\%). Of these 50 patients with Lower Urinary Tract stone disease, 45 were males i.e. $90 \%$ and only 5 were females i.e. $10 \%$.

441 patients having LUTS and BOO had Prostatic Hyperplasia or Enlargement i.e. 35\% of patients under study. Of these 441 patients, 375 patients i.e. $85 \%$ were more than 50 years of age and only 66 patients i.e. $15 \%$ were less than 50 year of age. Of all these 441 patients, 30 patients of BPH had reported with BOO after undergoing TURP (about 1.5 years back), only one patient was found to be having Bladder neck contracture i.e. only $3.3 \%$.

We selected patients of Haematurea based on finding of urine analysis and history of gross Haematurea. Patients having 3 or more RBC per HPF were leveled as having microhematuria and these giving history of frank haematurea were leveled as those with gross haematurea. 38 patients (out of 1252) reported gross haematurea i.e. $3 \%$ of patients. On cystoscopy only 4 cases (i.e. $10.5 \%$ ) had bladder tumor.

Out of 163 patients with Microscopic Hematuria (13\% of total patients) only 5 had bladder tumor (i.e. $3 \%$ of total patients.)

All these $9(4+5)$ cases of bladder tumor were more than 50 years old.

In 96 patients (7.7\% out of total of 1252 patients), who had undergone cystourethroscopy, there was evidence of inflammations in form of cystitis (Bladder) in 82 patients i.e. $85 \%$, prostatitis (Prostate)in 8 patients i.e. $7.7 \%$ and Urethritis (Urethra) in 6patients i.e.6.3\%.

In about 25 patients i.e. $2 \%$ of the patients undergoing cystourethroscopy had miscellaneous findings like bladder or urethral diverticulum, sacculations, urethral caruncle etc.

In 289 patients i.e. $23 \%$ of patients had normal cystourethroscopy findings. 


\section{Discussion}

This study was undertaken at our Hospital which is a district Hospital and poor patients from all over the State comes to us for treatment. Keeping this in mind to minimise the cost of treatment and investigation, cystourethroscopy was used as a diagnostic tool and also as an adjunct to various treatment modalities in patients coming with LUTS (both irritative and voiding), Haematurea, incontinence etc. We could avoid costly investigations like RGU/MCU, CT scan, MRI etc. by arriving at definitive diagnosis by doing Cystourethroscopy under Local anesthesia and we proceeded with definitive investigations and treatment only in cases where Cystourethroscopy result suggested so. We performed Cystourethroscopy on 1252 patients as per our inclusion and exclusion criterion. Out of 1252 patients, 150 patients i.e. $12 \%$ of the cases had stricture urethra, of these stricture cases $89 \%$ had anterior urethral stricture as compared to $11 \%$ patients having posterior urethral stricture. $94 \%$ of all these stricture patients were male.

50 patients i.e. $4 \%$ had stone in their lower urinary tract, majority of these stone casesi.e. $90 \%$ were found in urinary bladder. 441 patients i.e. $35 \%$ of the patients were found to be having prostatic hyperplasia $(\mathrm{BPH}) .85 \%$ of these $\mathrm{BPH}$ patients were above 50 years of age. Post TURP bladder Neck contracture was noticed only in $3.3 \%$ of patients undergoing this operation and coming back with complaints of BOO about 1.5 years after surgery.

201 patients i.e. $16 \%$ of Haematurea cases who underwent cystourethroscopy, bladder Tumor could be found only in 9 cases i.e. $4.5 \%$.

96 patient's ie. $7.7 \%$ patients had signs of inflammation in lower urinary tract, majority of them being cases of cystitis (82 out of 96 cases) i.e. $85 \%$ patients. Prostatitis was seen in only 8 patients out of 96 i.e. $7.7 \%$ and Urethritis in 6 patients i.e. $6.3 \%$. 25 patients out of 1252 i.e. $2 \%$ had miscellaneous findings.

289 patients i.e. $23 \%$ of patients examined had norm al CPE findings. These groups of patients were advised to undergo further investigations like UDS/VDU/CMG etc. to exclude neurogenic bladder, OAB etc.

\section{Conclusion}

Cystourethroscopy is a very useful diagnostic tool in cases of LUTS (both irritative and voiding), BOO, Haematurea and incontinence.

\section{Funding: None}

\section{Conflict of interest: None}

\section{References}

1. Gonzalez AN, Lipsky MJ, Li G, Rutman MP, Cooper KL, Weiner DM et al. The Prevalence of Bladder Cancer during Cystoscopy for Asymptomatic Microscopic Hematuria. Urology. 2019; 126:34-38

2. Aaronson DS, Walsh TJ, Smith JF, Davies BJ, Hsieh MH, Konety BR. Meta-analysis: does lidocaine gel before flexible cystoscopy provide pain relief? BJU Int. 2009; 104(4):506-9; discussion 509-10.

3. Okhunov Z, Hruby GW, Mirabile G, Marruffo F, Lehman DS, Benson MC et al. Prospective comparison of flexible fiberoptic and digital cystoscopes. Urology. 2009; 74(2):427-30.

4. Ramai D, Zakhia K, Etienne D, Reddy M. Philipp Bozzini (1773-1809): The earliest description of endoscopy. J Med Biogr. 2018; 26(2):137-141.
5. Bschleipfer T, Oelke M, Rieken M. [Diagnostic procedures and diagnostic strategy for lower urinary tract symptoms/benign prostatic hyperplasia: An overview]. Urologe A. 2019; 58(3):238-247.

6. Quayle SS, Ames CD, Lieber D, Yan Y, Landman J. Comparison of optical resolution with digital and standard fiberoptic cystoscopes in an in vitro model. Urology. 2005; 66(3):489-93.

7. Cadish LA, Ridgeway BM, Shepherd JP. Cystoscopy at the time of benign hysterectomy: a decision analysis. Am. J Obstet. Gynecol. 2019; 220(4):369.e1-369.e7.

8. Acar Ö, Tarcan T. Cystoscopic evaluation and clinical phenotyping in interstitial cystitis/bladder pain syndrome. J Turk GerGynecol Assoc. 2019; 20(2):117-122. [PMC free article]

9. Kurtz $\mathrm{M}$ et al. Etiology and evaluation of hematuria in adults, 2018. https://www.uptodate.com/contents/search. Accessed

10. Wein AJ et al. eds. Principles of urologic endoscopy. In: Campbell-Walsh Urology. 11th ed. Philadelphia, Pa.: Elsevier, 2016. https://www.clinicalkey.com. Accessed Aug. 8, 2018.

11. Fok CS et al. Diagnostic cystourethroscopy for gynecologic conditions, 2018. https://www.uptodate.com/contents/search. Accessed.

12. Gleason JL. Cystoscopy and other urogynecologic procedures. Obstetrics \& Gynecology Clinics of North America. 2013; 40:773.

13. Boyle P, Robertson C, Mazzetta C et al. (September). The prevalence of lower urinary tract symptoms in men and women in four centres. The UrEpik study. BJU Int. 2003; 92(4):409-14.

14. Immediate Versus Delayed Outpatient Flexible Cystoscopy: Final Report of a Randomized Study - HW Herr et al. Can J Urol. 2001; 8(6):1406-8. PMID 11788018. - Clinical Trial

15. Meta-Analysis: Does Lidocaine Gel before Flexible Cystoscopy Provide Pain Relief? - DS Aaronson et al. BJU Int. discussion 509-10. PMID 19239453. - Review. 2009; 104(4):506-9. 